Results Data will be collected by Dec 2015. Data cleaning and analysis will be completed by Mar 2016, and final results will be presented at Safety 2016.

Conclusions An important step to increasing bicycle helmet use is to determine additional factors, beyond laws, that may influence helmet use, such that social marketing campaigns can deliver targeted messages.

\section{PREVALENCE OF ALCOHOL AMONG CAR DRIVERS IN ROAD ACCIDENTS IN LATVIA: AN OVERVIEW FROM 2010 TO 2014}

Aija Bukova-Zideluna, Anita Villerusa. 'Riga Stradins University, Latvia

\subsection{6/injuryprev-2016-042156.849}

Background According to European Commission's data safety on Latvian roads is improving in recent years, however fatalities and casualties are still significantly higher in comparison with the situation in other countries. The aim of the study was to examine the prevalence of alcohol in blood samples from casualties in injury accidents (where at least one road vehicle in motion is involved, resulting in at least one injured or killed person) in Latvia from 2010 to 2014.

Methods Road safety accident database includes all the cases for which the police are informed. The cut-off concentrations for alcohol findings in blood samples were set according to the legislative limits (permissible alcohol level in blood is up to $0.5 \%$, but up to $0.2 \%$ for young road users having driving license for less than two years).

Results Out of 22332 persons injured in road accidents during this five year period, 965 were dead in 30 days after the accident. Alcohol consumption was tested for all persons involved in injury accidents. $7.2 \%$ of injured persons and $11.6 \%$ of killed persons in injury accidents were caused by drunk driving. Both victims of injured traffic accidents and accidents with alcohol involved were mostly males (60\%). The prevalence of alcohol was higher among persons killed twilight than daylight or night time. Drunk driver injured accidents have decreased during this five year period (2010-7.3\%; 2011-8\%; 2012-5.2\%; 2013-6\%; 2014$5.6 \%$ ) but it was not statistically significant.

Conclusions Alcohol still remains important contributing factor of traffic accidents in Latvia. In spite of legislation and public awareness campaigns, situation with drivers, who participate in road traffic under the influence of alcohol did not changes significantly during past years.

\section{LONGITUDINAL STUDY OF MOTOR VEHICLE CRASH RATES AMONG LICENSED TEEN DRIVERS WITH ADHD}

${ }^{1}$ Allison E Curry, ${ }^{1}$ Kristina B Metzger, ${ }^{1}$ Melissa R Pfeiffer, ${ }^{1}$ Flaura K Winston, ${ }^{2}$ Michael R Elliott, 'Thomas Power. 'The Children's Hospital of Philadelphia (CHOP), Philadelphia, Pennsylvania, USA; ${ }^{2}$ University of Michigan, Ann Arbour, Michigan, USA

\subsection{6/injuryprev-2016-042156.850}

Background Several small studies suggesting teens with ADHD are at heightened crash risk were conducted among more severely affected teens in highly specialised samples but had substantial methodological limitations. Thus, we conducted the first truly longitudinal study focused on comparing crash risk between teen drivers with and without ADHD. We also aimed to determine if the association between ADHD and risk varies by sex, licensing age, or over the course of licensure.

Methods We utilised electronic health records (EHR) to identify residents of New Jersey (NJ) born 1987-1995 who were patients of The Children's Hospital of Philadelphia's six NJ primary care practices within 4 years of driving-eligible age. EHR records were linked to NJ's state-wide driver licensing and crash databases through June 2012. Subjects were classified as having ADHD using ICD-9-CM diagnosis codes and known chronic conditions from their EHR. Cox regression was used to estimate adjusted hazard ratios (HR) to compare crash rates for 1,307 licensed teens with and 10,415 licensed teens without ADHD.

Results Subjects had a median [interquartile range] of 17 [9, 28] CHOP primary care visits, were $18[16,19]$ years old at their last visit, and were $21[19,23]$ years old at study end. Overall, the crash rate for teens with ADHD was 35\% higher (95\% CI: 1.22, 1.49) than for teens without ADHD. Modelling revealed heightened risk for male teens (HR [95\% CI]: 1.43 [1.27, 1.61]) with less evidence of an increase among females (1.17 [0.97, 1.42]). Conversely, the association between ADHD and crash involvement did not vary by licensing age or over time.

Conclusions Young novice drivers with ADHD-and in particular males-appear to be at increased crash risk, although the estimated increase is notably lower than frequently cited figures from previous small studies of self-reported crashes. Additional research is needed to understand the specific mechanisms by which ADHD influences per-driver crash risk.

\section{CHILDREN \& ROAD SAFETY- A MULTIPRONGED APPROACH}

Rupa Kothari, G Madhavi. Safe Kids Foundation, India

\subsection{6/injuryprev-2016-042156.851}

Background 'Safe Kids Foundation's, Walk This Way'-a pedestrian safety program teaches children to 'Walk Safe and Cross Safe' through educational sessions and awareness activities. Annually more than 220,000 children from 350 schools in Mumbai, Delhi and Ahmedabad are benefited by this program. While educating children on road safety, it was observed that children were receptive, willing to adapt to road safety rules and thereby act as 'young advocates' to facilitate behaviour change.

Objective To train teachers and build their capacities to carry out age specific innovative interventions on road safety in schools. To conscientize students, parents communities and other stakeholders on the issues of road safety through various activities in school/communities to naturally build the safety culture amongst the targeted stakeholders. To conduct research to study impact of educational sessions on children.

Results Teachers, children, parents and communities are conscientized on road safety. In this model, to reinforce the messages at regular intervals, activities like game, songs, competitions are conducted. Educational tools such as flipcharts, posters, banners, demonstrations materials like dummy signals, zebra crossing, activity books etc, help in better understanding and comprehension of the information provided. Peer to peer education enabled sensitise 2,500 children by fellow mates. Children's initiative as safety advocates helped in effectively bringing attitudinal \& behavioural changes amongst children and adults. Photo voice, model school zone are research based interventions, initiatives like observing Global Road Safety Week, Road Safety Week- India 